\title{
RETINA FUNDUS IMAGE MASK GENERATION USING PSEUDO PARAMETRIC MODELING TECHNIQUE
}

\author{
A.M. Aibinu, M.J.E. SAlami AND A.A. Shafie \\ Department of Mechatronics Engineering, \\ Faculty of Engineering, International Islamic University Malaysia \\ P. O. Box 10, 50728, Malaysia. \\ E-mail: maibinu@iium.edu.my
}

\begin{abstract}
This paper discusses a new pseudo modeling technique for the generation of retina fundus image (RFI) mask. The model coefficients necessary for the generation of the mask has been estimated from the synaptic weights of real-valued neural network. Performance analysis of the newly proposed three step technique has been evaluated using DRIVE databases and other RFI obtained from other sources. he accuracy obtained by the application of the proposed technique on RFI contained in the DRIVE database varies between $99.62 \%$ and $99.97 \%$.
\end{abstract}

KEYWORDS: Diabetes, Parametric Modeling Technique, Real-Valued Neural Network (RVNN), Retina Fundus Image

\section{INTRODUCTION}

In recent times, there have been a tremendous increase in age and society related diseases such as diabetes. In Sweden, $4 \%$ of the country population have been diagnosed of diabetes [1] while approximately $2 \%$ of the working class in United Kingdom are diabetic patient [2]. Similar study in Malaysia shows a steady rise in the population of diabetic patient from $0.65 \%$ in 1960 to $2 \%$ in 1980 [3] and according to America Diabetes Association, $6.3 \%$ of the total population of Americans have been diagnosed of this disease [4]. Also, it have been estimated that over $13 \%$ of the Egyptian with age above 20 years will have diabetes by the year $2025[5,6]$ and about $5.5 \%$ of the world population have been diagnosed of the disease $[6,7]$.

Diabetes can be described as a disorder in body metabolism. Digested food enters the body stream with the aid of a hormone called insulin which is produced by the pancreas to automatically produce the correct amount of insulin needed for allowing glucose absorption from the blood into the cells. For individuals with diabetes, the pancreas either produces too little or no insulin or the cells do not react properly to the insulin that is produced by the body. The build up of glucose in the blood, overflows into the urine and then passes out of the body. Therefore, the body loses its main source of fuel even though the blood contains large amounts of glucose [8]. 
The effect of diabetes on the eye is known as Diabetic Retinopathy (DR) and this can be loosely classified into : Background Diabetic Retinopathy (BDR), Proliferate Diabetic Retinopathy (PDR) and Severe Diabetic Retinopathy (SDR) [9, 10, 11, 12]. If DR is left untreated, the disease can degenerate and lead to blindness. According to World Health Organization (WHO) report released in 2002, $8.7 \%$ of the population of blindness have been linked to DR and taking a micro look at United State alone, about $8 \%$ of the blindness for the age bracket 20 to 74 have been due to DR $[4,13]$.

Early detection, diagnosis and treatment have been identified as one of the ways to achieve reduction in the percentage of visual impairment caused by diabetes [1,9]. Different approaches have been suggested for early detection and monitoring of the DR such as the use of computer aided automatic diagnosis of DR from retina fundus image (CAADRFI). CAADFRI and its variants (as suggested by others researchers) have the ability to reduce the number of blindness due to DR by almost $50 \%$ and they provide considerable time saving for patient and ophthalmologists, reduce wears and tears on equipments [6, 11-12]. However, most of the acquired RFI usually have severe limitation such as quality, acquisition and problems. In ensuring that screeners and ophthalmologists have good view of the whole retina fundus image (RFI), more than the needed number of images are sometimes acquired. The existence of good image processing algorithm that can improve and consistently guarantee good image quality will lead to a drastic reduction in the RFI acquisition and storage space requirements since only a sufficient number of images will be saved [5, 9-10].

Various algorithms have been developed by various researchers for the CAADRFI system, the effectiveness of which greatly depends on the quality of the acquired RFI [221]. Most of these techniques can be regarded as a three-stage technique, namely RFI preprocessing stage so as to solve the problems of RFI mask generation, object motion, poor lighting and illumination problems; image segmentation stage and diseases classification for the anatomy detection and classification.

A typical RFI used for CAADFRI is shown in Fig. 1a, the whole regions contained in this RFI can be grouped into two main classes, namely the white semi-circular region of interest (ROI) and the black background [6]. Figure 1b is subsequently referred to in this paper as RFI mask. The process of generating the RFI mask involves labeling pixel belonging to the ROI as one group and those belonging to the surrounding areas of the ROI as background. Incorrect delineation of background from ROI greatly affects the results of the CAADFRI system hence the need for accurate delamination of ROI from the background in RFI for a CAADFRI system. A typical RFI mask is shown in Fig. 1b.

Different methods of generating RFI mask have been suggested in the literature however in this paper, a new method of generating RFI mask using pseudo parametric modeling approach is presented. Review of related work is discussed in section 2 while the newly proposed technique is presented in section 3. Performance analysis of the proposed technique and conclusion are contained in section 4 and section 5 respectively. 


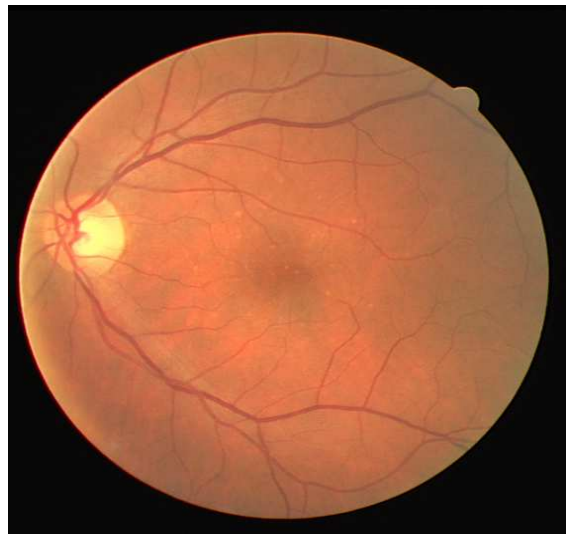

Fig. 1: (a)Retina Fundus Image (RFI)

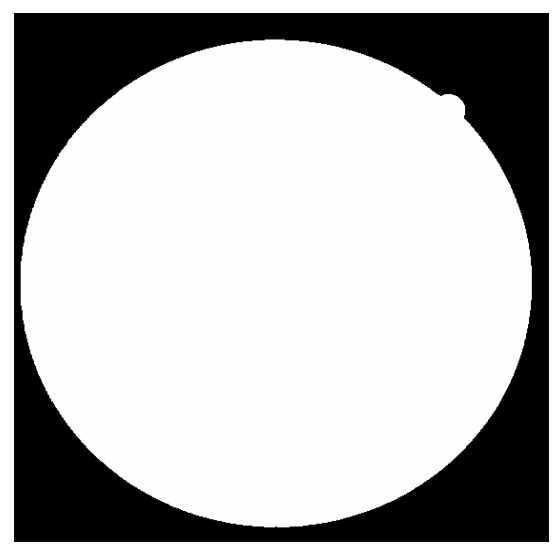

(b) Retina Fundus Image (RFI) mask.

\section{LITERATURE REVIEW}

A comparative performance measure applicable to the three major RFI preprocessing steps namely: mask generation, illumination equalization and color normalization has been reported in [6]. Three methods of automatic mask generation was evaluated in the work, and it was observed that using morphological operators on the thresholded red band result in superior performance over either the simple thresholding of the green band proposed by Goatman et al. [2] or thresholding on the the three RGB channels proposed in [19].

In a related work by Gagnon et al. [19], several methods of detecting anatomical structures in RFI were presented. The algorithm proceed through five main steps which involve: automatic mask generation using pixels value statistics and color threshold; visual image quality assessment using histogram matching and Canny edge distribution modeling; optic disk localization using pyramidal decomposition, Hausdorff-based template matching and confidence assignment; Macula localization using pyramidal decomposition and vessel network tracking using recursive dual edge tracking and connectivity recovering. Results obtained in RFI mask generation stage show that the proposed technique is robust against low visual quality of the images and is independently of the position of the Macular optic disk-centered when detecting the optic disk. Success rates of $100 \%$ is reached for the Optic disk detection and $95 \%$ for the Macula detection.

Early detection of DR from RFI has been proposed in [20]. RFI model was developed based on probability distribution function of Macular pigment, haemoglobin and melanin to represent macular region, retinal vasculature and background, respectively. Independent component analysis based on the spectral absorbance of the model was then applied to determine retinal pigments from RFI. The proposed technique outperforms other noninvasive enhancement methods, such as contrast stretching, histogram equalization and contrast limited adaptive histogram equalization techniques, however little or no mention was made of methods of RFI mask generation in this work.

In another related work, an expert system for early diagnosis of signs of DR was presented [21]. The process starts with an extraction of sub-image containing the optic 
disk (OD) followed by OD boundary extraction in parallel from both the red and green channels of this sub-image by means of morphological and lastly the edge detection techniques was then applied. Both OD boundaries are approximated by a circumference using the circular Hough transform. The location procedure succeeded in $99 \%$ cases, taking an average computational time of $1.67 \mathrm{~s}$. with a standard deviation of $0.14 \mathrm{~s}$ while the segmentation algorithm rendered an average common area overlapping between automated segmentations and true OD regions of $86 \%$ with an average computational time of $5.69 \mathrm{~s}$ having $0.54 \mathrm{~s}$ standard deviation.

In [14], a computer-aided diagnosis (CAD) system that facilitates the interpretation of RFI was developed. In this technique, RFI was converted into a feature vector that was created using histograms obtained from multiple images with different resolutions. An accuracy of about $96.34 \%$ was obtained with this technique. Similarly, Kee et. al developed a computer based approach for automatic segmentation of blood vessels in screening large numbers of vessel abnormalities contained in RFI [14]. Although the results in this proposed method were compared to a manually segmented image but no empirical interpenetration of the accuracy was given.

A three-stage framework for detection of vascular intersection namely bifurcationpoint (BP) and cross-point in RFI using a hybrid combined cross-point number (CCN) approach was proposed in $[11,12]$. Using a receiver operator curve (ROC) based analysis on the proposed hybrid algorithm, a very high precision and true positive rate with a very small false error rate were obtained, indicating an improved performance when compared to the individual performance of modified cross-point number (MCN) and Simple cross point number proposed in $[12,16]$. Another version to the use of $5 \times 5$ windowed based $\mathrm{CCN}$ was suggested in [11, 12] and also proposed in [16]. The hybrid technique was recently modified by using a 7x7 window in detecting vascular intersection in RFI [16]. The newly modified technique was tested on 100 RFI as opposed to the test performed in [11] on two publicly available RFI images databases.

Detection of abnormalities related to the inner structures of RFI using image enhancement, Max-Tree image representation and filtering procedure was proposed in [18]. This method produced an accuracy of $93.95 \%$ and $94.21 \%$ in detecting associated abnormalities on 40 and 20 RFI respectively.

Detection of the macula in the processing of RFI was proposed in [13]. Regions of dark spots were detected by finding the coordinates with the lowest pixel intensity and determining the average pixel neighborhood intensities and these were ranked to obtain the region containing the macula. In evaluating the performance of this technique, a total of 162 images (81 RFI of left eye and 81 RFI of right eye) were used and $98.8 \%$ accuracy was obtained when the algorithm was applied on high quality RFI. However, this proposed technique was unable to accurately detect the macula in a low quality RFI.

Edge sharpening technique is employed in highlighting the boundary of RFI in the development of automatic detection of lesions in RFI [3]. Also, automatic detection and extraction of exudates and optic disk in RFI was proposed in [1]. The RFI was preprocessed in order to improve the contrast and the overall color saturation in the image, then the optic disk was eliminated and the exudates were segmented using spatially 
weighted Fuzzy c-means clustering. The proposed algorithm for optic disk detection produced $92.53 \%$ accuracy. The sensitivity and the specificity of the proposed algorithm for exudates detection were $86 \%$ and $98 \%$ respectively.

Development of an automatic measurement and analysis of vessel tortuosity in color RFI was proposed in [35]. Two sets of RFI for normal and diabetic persons were considered in this work and the proposed approach will be suitable for predicting or earlier diagnosis of diabetes or cardiovascular diseases. It was observed that higher vessel tortuosity appears in diabetic persons but this requires confirmation in studies with detailed clinical information and adequate sample size.

Villalobos and Riveron in [36] presented a fast automatic retinal vessel segmentation and vascular landmarks extraction method for biometric applications using a three stage approach namely preprocessing, main processing and postprocessing steps. Application of this technique on images in DRIVE database, shows that this proposed method can a accurately detect and extract information from a RFI.

\section{DEVELOPMENT OF PSEUDO INTELLIGENT TECHNIQUE FOR RFI}

Parametric modeling technique involves representation of data in an efficient and parsimonious form using minimum adjustable parameters [22-27]. The steps involved are model selection, model parameters determination and model validation. Model selection is basically about choosing an appropriate parametric form for the signal or data to be modeled and some of the known models include autoregressive (AR) model, moving average (MA) model, autoregressive moving average (ARMA) model and their variants (such as autoregressive with external input (ARX) model, vector autoregressive (VAR), autoregressive moving average with external inputs (ARMAX) model, autoregressive integrated moving average (ARIMA) model, autoregressive integrated moving average model etc) [22-26]. Similarly, model parameters determination is concerned with the use of computationally efficient algorithm to determine model parameters while model validation involves the evaluation of how well the selected model captures the key features of the modeled data using the estimated model parameters [22-27].

In this work, new method of generating RFI mask using RVNN is proposed. Detailed knowledge about RVNN can be obtained from [28, 29] and differences between a RVNN and its counterpart, the complex valued neural network can be obtained from [30-34]. The required model coefficients are obtained from the synaptic weights and the adaptive coefficients of the activation functions in a trained RVNN.

Consider the RFI acquired in Red-Green-Blue (RGB) mode shown in Figure 1a, with the mask shown in Figure 1b, the image consist of three components, namely red $(R)$, green $(\mathrm{G})$ and blue (B) channels. These components fuse to give the final image shown in Fig. 1a using the RGB scheme depicted in Fig. 2. 


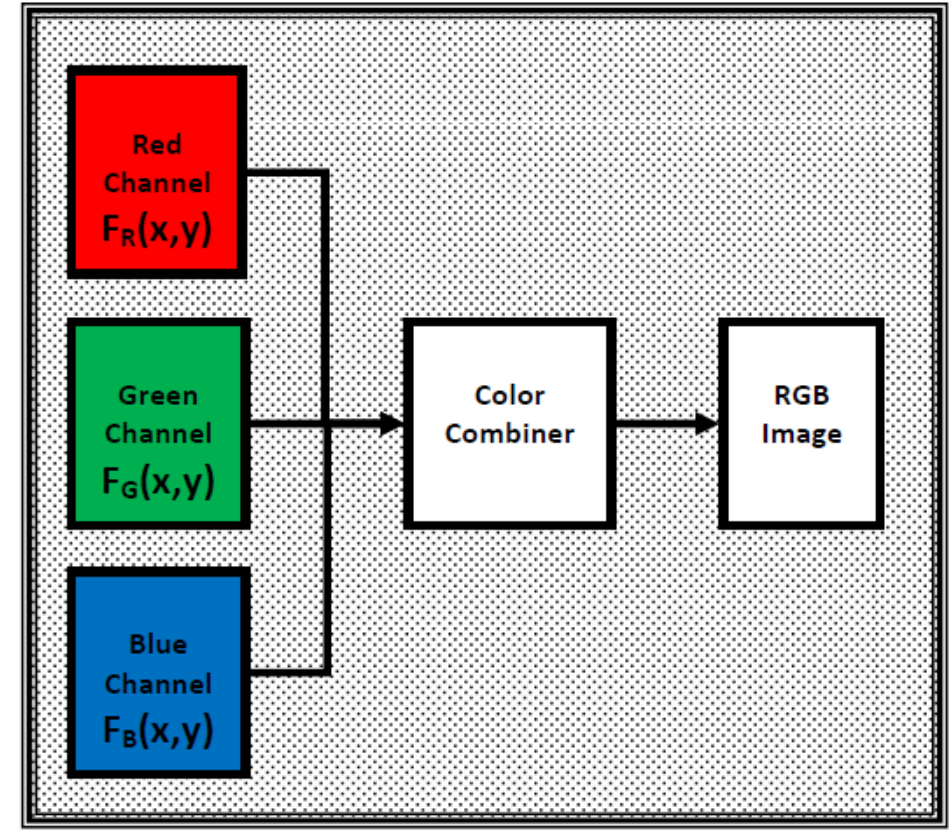

Fig. 2: RGB color Scheme Generation as applicable to RFI.

Since the final image $F(x, y)$ can be represented by its components color as

$$
F(x, y)=a_{R} F_{R}(x, y)+a_{G} F_{G}(x, y)+a_{B} F_{B}(x, y)
$$

where $a_{R}, a_{G}$ and $a_{B}$ are the coefficients required to generate the RFI mask (Fig. 1b).

Similarly, consider the AR model shown in Fig. 3, which is driven by a white noise sequence $x(n)$ to produce an output sequence $y(n)$ according to

$$
\begin{aligned}
y(n) & =-\sum_{k=1}^{p} a_{k} y(n-k)+b_{0} x(n) \\
& =-\left[a_{1} y(n-1)+a_{2} y(n-2)+\ldots+a_{p} y(n-p)\right]+b_{0} x(n)
\end{aligned}
$$

where $a_{k}, 1 \leq k \leq p$ and $p$ are the AR model coefficients and model order respectively.

By comparing (1) and (2), the problem of generating the required RFI mask reduces to that of estimating the required coefficients in (1), thus the problem reduces to a pseudo parametric modeling approach. Hence, a new three stage pseudo parametric modeling approach for the determination of the model coefficients $a_{R}, a_{G}$ and $a_{B}$ from the synaptic weights and adaptive activation function coefficients of a RVNN is shown in Fig. 4. 


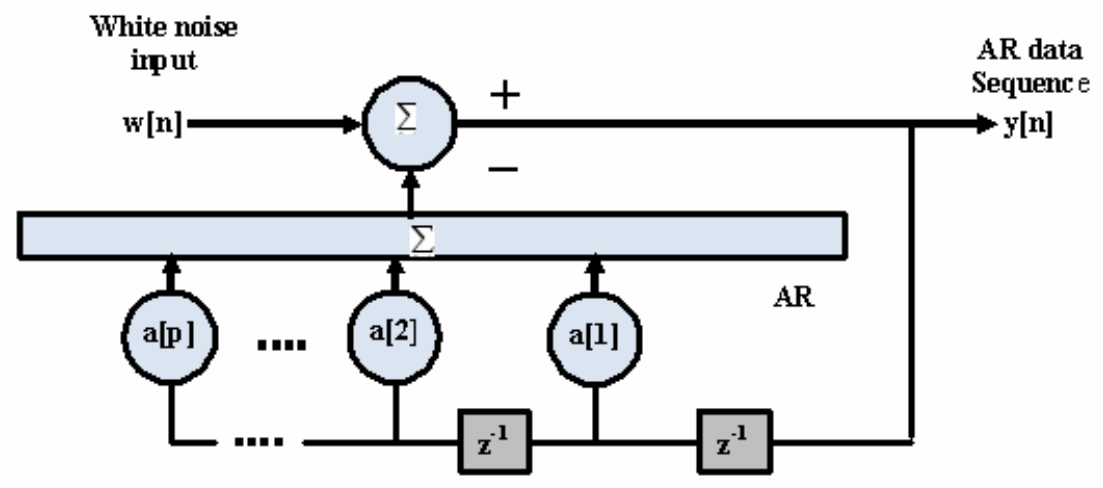

Fig. 3: AR model representation.

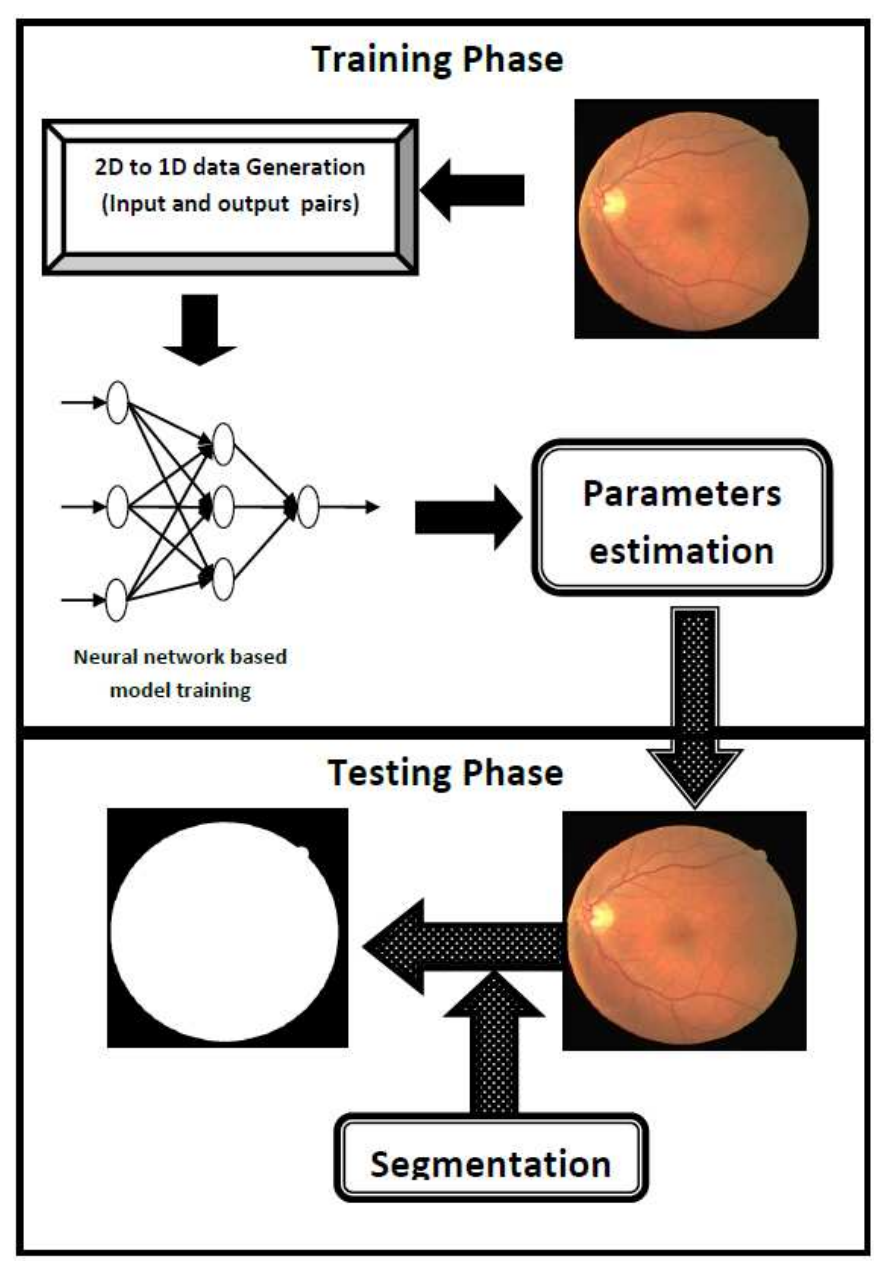

Fig. 4: RVNN-based pseudo AR model RFI mask generation. 
By considering the RVNN-based RFI mask generation shown in Figure 5, the output $y(n)$ is expressed as

$$
y(n)=\alpha \hat{\mathbf{o}}\left(\sum_{l=1}^{M} w_{l 1}\left(\beta_{l} \hat{o}\left(\sum_{k=1}^{p} v_{k l} y_{k}(n)+g_{0 l}\right)\right)+h_{01}\right)
$$

where $M$ is the number of neurons in the hidden layer, $w_{l 1}$ is the weight connecting node $l$ in the hidden layer to output layer, $h_{0^{1}}$ is the bias term of output neuron, $\vartheta_{l}$ is the $l^{\text {th }}$ output of the hidden node $l, \alpha$ is the adaptive coefficient of the linear output activation function, $v_{k l}$ is the weight connecting input node $k$ to hidden node $l, g_{l}$ is the bias of the hidden node $l, \beta_{l}$ is the adaptive coefficient of hidden node linear activation function and $P$ is the number of input nodes. For an RGB-based RFI, $P=3$.

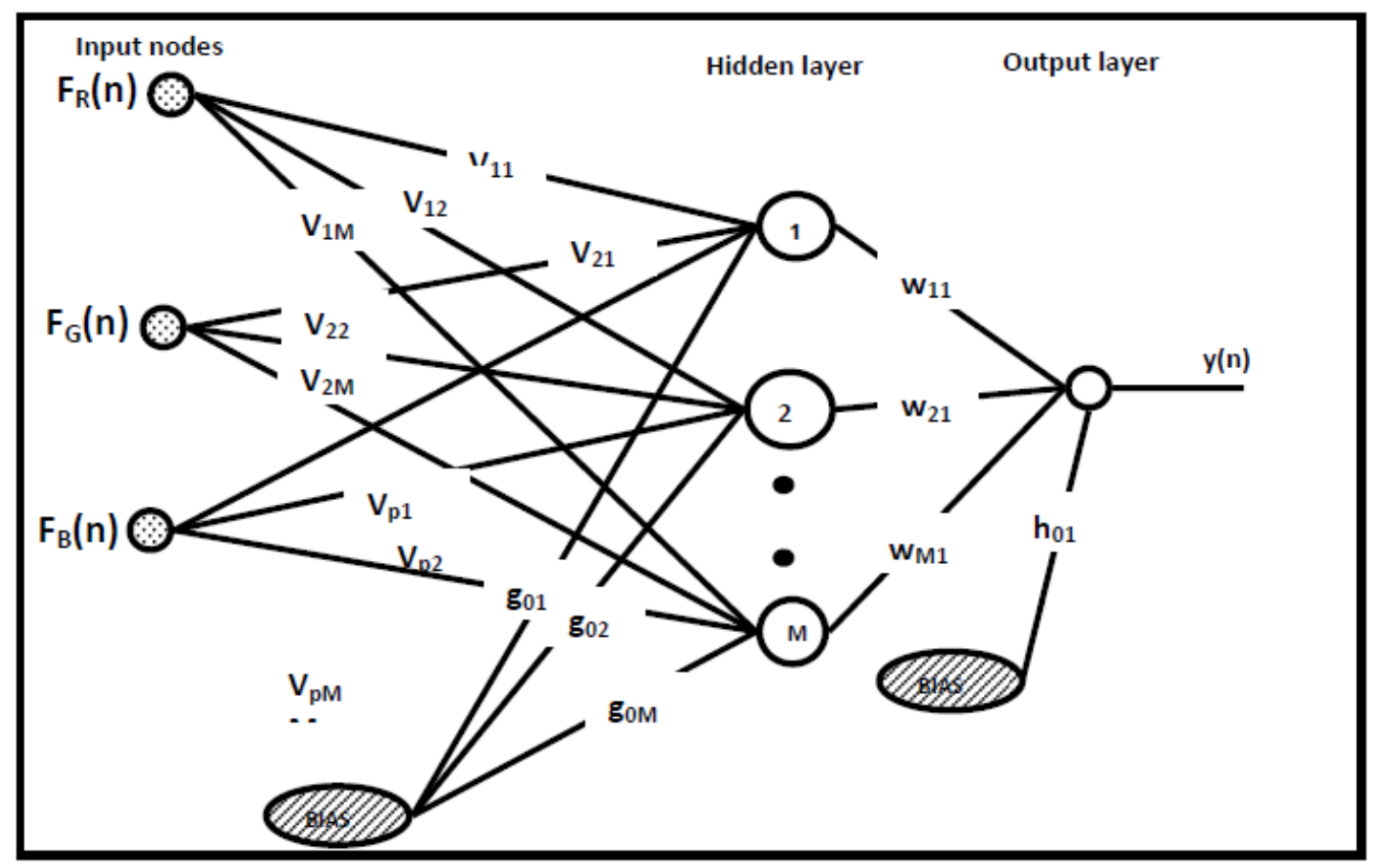

Fig. 5: RVNN-based RFI mask neural network diagram.

Using linear transfer activation functions in both the hidden and output layer (i.e ô() is the activation function), the required RFI mask generation coefficients are given as

$$
a_{k}=\alpha \sum_{l=1}^{M} w_{l l} v_{k l} \beta_{l}, \quad 1 \leq k \leq 3
$$


Thus, $a_{1}, a_{2}$ and $a_{3}$ are the model coefficients for the R, G and B components of the RGB-based RFI respectively and the coefficients obtained in (4) are equivalent to the required coefficients $a_{R}, a_{G}$ and $a_{B}$ given in (1).

\section{PERFORMANCE ANALYSIS}

The evaluation of the proposed technique is presented in this section. Performance evaluation of this technique has been carried out using the digital retinal images for vessel extraction popularly known as the DRIVE database. The images in the database were acquired using a Canon CR5 non-mydriatic 3CCD camera with a 45 degree field of view (FOV). The dataset contains 40 RFI of $584 \times 565$ pixels and the images have been divided into a training set consisting of 20 RFI and a test set of 20 RFI [37]. In this proposed technique only one single image is enough for training of the proposed RVNNbased pseudo modeling technique and the remaining 39 images can be used for testing. In evaluating the performance of this technique some of the mathematical definitions and acronyms used include:

$$
\begin{gathered}
\text { Accuracy }(A C C)=\frac{T P+T N}{P+N} \\
\text { Precision }(P R)=\frac{T P}{T P+F P} \\
\text { False Positive Rate }(F R)=\frac{F P}{P} \\
\text { True Positive Rate }(T P R)=\frac{T P}{\text { Total Positives }}
\end{gathered}
$$

where TP, TN, FP and FN represent respectively true positive, true negative, false positive and false negative[38].

In the training phase, a single RFI is used to generate the training data, sample of the ROI and the background serve as the input to the RVNN. The ROI is assigned a target value of 1 while the background is assigned a target value of 0 . The input and the target data are concatenated and normalized separately and these are then fed to the RVNN. Once the RVNN converged, the synaptic weights and adaptive coefficients are extracted and the required coefficients could be computed. In the testing mode, the obtained pseudo modeling coefficients are applied on the input image, the output is then clustered into two distinct classes, the ROI and the background.

In this paper, two types of clustering techniques have been evaluated, namely, the simple thresholding and k-means algorithm. one RFI image in the DRIVE database have 
been used for the extraction of the mask coefficients, from which the value of the required coefficients obtained are $-2.7677,0.5609,-0.5417$ for $a_{R}, a_{G}$ and $a_{B}$ respectively.

Results obtained from the application of these coefficients on RFI contained in DRIVE database are discussed herein. Each experiment has been repeated 30 times and the average accuracy value was then computed, the RFI mask obtained from the use of this technique was compared with the mask given in the DRIVE database and performance measurement criteria discussed earlier were then computed.

Table 1 shows the results obtained by the application of the obtained coefficients and simple thresholding technique on one of the RFI from the database while Table 2 depicts the results obtained by the use of k-means algorithm. As observed from the table, the use of simple thresholding of the RVNN output performs far better than that of k-means algorithm, though the performance of the simple thresholding technique reduces at very low or high threshold values.

Table 1: RFI mask generation using Simple thresholding clustering technique.

\begin{tabular}{|c|c|c|c|c|}
\hline Threshold & Accuracy & Precision & TPR & FPR \\
\hline 0.0500 & 0.7074 & 0.7023 & 1.0000 & 0.9443 \\
\hline 0.1000 & 0.9433 & 0.9240 & 1.0000 & 0.1831 \\
\hline 0.1500 & 0.9954 & 0.9933 & 1.0000 & 0.0149 \\
\hline 0.2000 & 0.9996 & 0.9995 & 1.0000 & 0.0012 \\
\hline 0.2500 & 0.9995 & 1.0000 & 0.9993 & 0.0000 \\
\hline 0.3000 & 0.9990 & 1.0000 & 0.9986 & 0.0000 \\
\hline 0.3500 & 0.9985 & 1.0000 & 0.9978 & 0.0000 \\
\hline 0.4000 & 0.9981 & 1.0000 & 0.9972 & 0.0000 \\
\hline 0.4500 & 0.9976 & 1.0000 & 0.9966 & 0.0000 \\
\hline 0.5000 & 0.9971 & 1.0000 & 0.9958 & 0.0000 \\
\hline
\end{tabular}

Table 2: RFI mask generation using K-means clustering technique.

\begin{tabular}{|c|c|c|c|}
\hline Accuracy & Precision & TPR & FPR \\
\hline 0.9916 & 1.0000 & 0.9878 & 0.0000 \\
\hline 0.9916 & 1.0000 & 0.9878 & 0.0000 \\
\hline 0.9916 & 1.0000 & 0.9878 & 0.0000 \\
\hline 0.9916 & 1.0000 & 0.9878 & 0.0000 \\
\hline 0.9916 & 1.0000 & 0.9878 & 0.0000 \\
\hline 0.9916 & 1.0000 & 0.9878 & 0.0000 \\
\hline 0.9916 & 1.0000 & 0.9878 & 0.0000 \\
\hline
\end{tabular}




\begin{tabular}{|l|l|l|l|}
\hline 0.9916 & 1.0000 & 0.9878 & 0.0000 \\
\hline 0.9916 & 1.0000 & 0.9878 & 0.0000 \\
\hline 0.9916 & 1.0000 & 0.9878 & 0.0000 \\
\hline
\end{tabular}

Table 3 shows the results obtained by the application of the obtained coefficients on all the RFI contained in DRIVE training database. The accuracy of the k-means clustering technique is compared with that of simple thresholding technique over all the RFI contained in the training subset. From the results shown in this table, the use of simple thresholding technique outperforms that of k-means algorithm.

Table 3: Comparing K-means clustering with simple thresholding (with 0.20 threshold value) for RFI mask generation.

\begin{tabular}{|c|r|r|r|r|r|r|}
\hline \multirow{2}{*}{$\begin{array}{c}\text { Image } \\
\text { Number }\end{array}$} & \multicolumn{3}{|c|}{ K-means } & \multicolumn{3}{c|}{ Simple Threshold } \\
\cline { 2 - 7 } & Accuracy & Precision & \multicolumn{1}{c|}{ TPR } & Accuracy & Precision & TPR \\
\hline 21 & 0.9990 & 1.0000 & 0.9985 & 0.9934 & 0.9904 & 1.0000 \\
\hline 22 & 0.9914 & 1.0000 & 0.9876 & 0.9997 & 0.9996 & 1.0000 \\
\hline 23 & 0.9915 & 1.0000 & 0.9878 & 0.9996 & 0.9994 & 1.0000 \\
\hline 24 & 0.9916 & 1.0000 & 0.9878 & 0.9996 & 0.9995 & 1.0000 \\
\hline 25 & 0.9907 & 1.0000 & 0.9864 & 0.9996 & 0.9995 & 1.0000 \\
\hline 26 & 0.9992 & 1.0000 & 0.9988 & 0.9934 & 0.9905 & 1.0000 \\
\hline 27 & 0.9921 & 1.0000 & 0.9886 & 0.9997 & 0.9995 & 1.0000 \\
\hline 28 & 0.9924 & 1.0000 & 0.9889 & 0.9997 & 0.9995 & 1.0000 \\
\hline 29 & 0.9875 & 1.0000 & 0.9819 & 0.9996 & 0.9994 & 1.0000 \\
\hline 30 & 0.9931 & 1.0000 & 0.9900 & 0.9996 & 0.9994 & 1.0000 \\
\hline 31 & 0.9919 & 1.0000 & 0.9883 & 0.9996 & 0.9994 & 1.0000 \\
\hline 32 & 0.9992 & 1.0000 & 0.9989 & 0.9916 & 0.9879 & 1.0000 \\
\hline 33 & 0.9923 & 1.0000 & 0.9888 & 0.9997 & 0.9995 & 1.0000 \\
\hline 34 & 0.9701 & 1.0000 & 0.9565 & 0.9972 & 0.9959 & 1.0000 \\
\hline 35 & 0.9913 & 1.0000 & 0.9874 & 0.9997 & 0.9995 & 1.0000 \\
\hline 36 & 0.9928 & 1.0000 & 0.9895 & 0.9996 & 0.9995 & 1.0000 \\
\hline 37 & 0.9924 & 1.0000 & 0.9890 & 0.9997 & 0.9995 & 1.0000 \\
\hline 38 & 0.9963 & 1.0000 & 0.9946 & 0.9961 & 0.9943 & 1.0000 \\
\hline 39 & 0.9930 & 1.0000 & 0.9898 & 0.9997 & 0.9995 & 1.0000 \\
\hline 40 & 0.9922 & 1.0000 & 0.9887 & 0.9995 & 0.9993 & 1.0000 \\
\hline & & & & & & \\
\hline
\end{tabular}

Furthermore, in another experiment using RFI acquired from another hospital in Sweden [9-10], the coefficients obtained previously from DRIVE database was applied on the RFI from [9-10], result obtained is shown in Figure 6. The unavailability of the ground truth mask leads to only subjective evaluation of the obtained mask. 


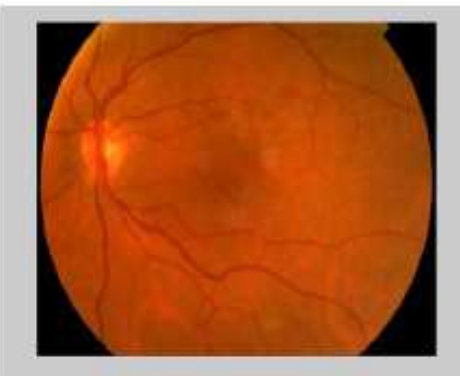

(a)

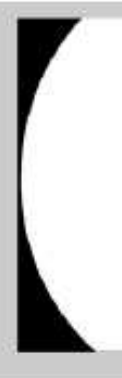

(b)

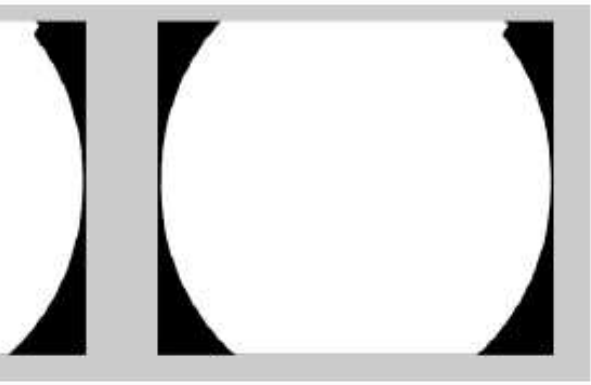

(c)

Fig. 6: (a) Original RFI, (b) RFI mask using k-means clustering technique, (c) RFI mask using simple thresholding technique.

Lastly, the performance of the proposed pseudo modeling algorithm was evaluated on RFI acquired from another fundus camera in Malaysia, result obtained is shown in Figure 7. The unavailability of the ground truth mask leads to only subjective evaluation of the obtained mask. The superior performance of the simple thresholding technique is depicted in the resulting images $7 \mathrm{~b}$ and $7 \mathrm{c}$.

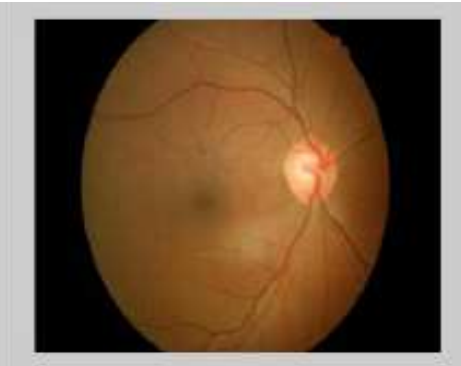

(a)

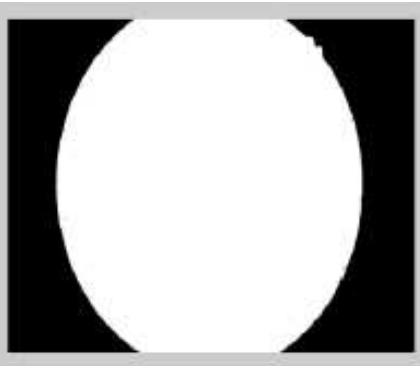

(b)

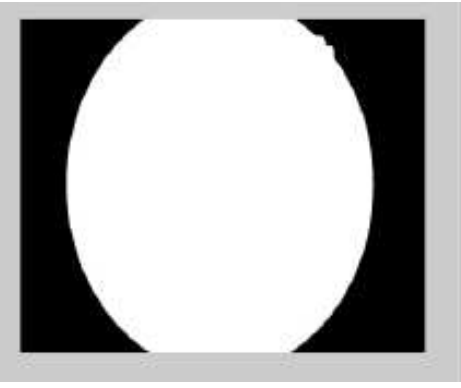

(c)

Fig. 7: (a) Original RFI, (b) RFI mask using k-means clustering technique, (c) RFI mask using simple thresholding technique.

\section{CONCLUSION}

A new method of extracting RFI using pseudo modeling technique has been presented in this paper and several existing works on CAADRFI have been discussed herein. The accuracy obtained by the application of the proposed technique on RFI contained in the DRIVE database varies between $99.62 \%$ and $99.97 \%$. It has also been shown that using a simple thresholding to classify the output of the RVNN technique leads to an improved performance as compared to the use of k-means clustering technique. Furthermore, the proposed technique has also been used to generate RFI mask from low visual quality RFI using the same extracted pseudo model coefficients. 


\section{ACKNOWLEDGMENT}

This work is partially supported by Malaysian E-science grant $01-01-08-S F 0083$.

\section{REFERENCES}

[1] Conference Report: Screening for Diabetic Retinopathy in Europe 15 years after the St. Vincent declaration the Liverpool Declaration 2005. Retrieved March 18, 2010, From website: http://reseauophdiat.aphp.fr/Document/Doc/confliverpool.pdf\#search='www.drsceening 2005.org.uk'

[2] K. A. Goatman, A. D. Whitwam, A. Manivannan, J. A. Olson, and P. F. Sharp, Colour normalisation of retinal images, Proc. Med. Imag. Understanding and Analysis, 2003.

[3] H. Yazid, H. Arof and N. Mokhtar, Edge Sharpening for Diabetic Retinopathy Detection, IEEE Conference on Cybernetics and Intelligent Systems, pp 41-44, 2010.

[4] P. Kahai, K. R. Namuduri and H. Thompson, A Decision Support Framework for Automated Screeing of Diabetic Retinopathy, Int. Journal of Biomedical Imaging, pp 1-8, Volume 2006, Article ID 45806.

[5] W. H. Herman, R. E. Aubert, M. A. Ali, E. S. Sous, and A. Badran, Diabetes mellitus in Egypt: risk factors, prevalence and future burden, Eastern Mediterranean Health J., (3), 144-148, 1997.

[6] A. A. A. Youssif, A. Z. Ghalwash, and A. S. Ghoneim, A Comparative Evaluation of Preprocessing Methods for Automatic Detection of Retinal Anatomy, Proceedings of the Fifth International Conference on Informatics and Systems (INFOS 07), pp 24 -30, March 24-26, 2007.

[7] J. Staal, M. D. Abràmoff, M. Niemeijer, M. A. Viergever and B. van-Ginneken, Ridge-based vessel segmentation in color images of the retina, IEEE Trans. Med. Imag.,(23), 501-509, April 2004.

[8] Abate Diabetes: Diabetes. Accessed March 21, 2006, from Website: http://www.abatediabetes.com/diabetes.html

[9] A. M. Aibinu, M. I. Iqbal, M. Nilsson and M. J. E. Salami, Automatic Diagnosis of Diabetic Retinopathy from Fundus Images Using Digital Signal and Image Processing Techniques, International Conference on Robotics, Vision, Information, and Signal Processing, Penang, Malaysia, pp. 510 - 515, Nov. 2007.

[10] A. M. Aibinu, M. I. Iqbal, M. Nilsson and M. J. E. Salami, A New Method of Correcting Uneven Illumination Problem in Fundus Images, International Conference on Robotics, Vision, Information, and Signal Processing, Penang, Malaysia, pp. 445 - 449, Nov.2007.

[11] A.M. Aibinu, M.I. Iqbal, A.A. Shafie, M.J.E. Salami, M. Nilson, Vascular intersection detection in retina fundus images using a new hybrid approach, Computers in Biology and Medicine, (40), 81-89, January, 2010.

[12] M. I. Iqbal, A. M. Aibinu, M. Nilsson, I. B. Tijani, and M. J. E. Salami, Detection of Vascular Intersection in Retina Fundus Image Using Modified Cross Point Number and Neural Network Technique, Proceedings of the International Conference on Computer and Communication Engineering, pp, 241-246, 2008. 
[13] N. M. Tan, D.W.K. Wong, J. Liu, W.J. Ng, Z. Zhang, J.H. Lim, Z. Tan, Y. Tang, H. Li, S. Lu and T.Y. Wong, Automatic Detection of the Macula in the Retinal Fundus Image by Detecting Regions with Low Pixel Intensity, International Conference on Biomedical and Pharmaceutical Engineering, ICBPE '09, pp 1-5, 2-4 Dec. 2009, Singapore.

[14] S. Lu, J. Liu, J. H. Lim, Z. Zhang, T. N. Meng, W. K. Wong, H. Li, and T. Y. Wong, Automatic Fundus Image Classification for Computer-Aided Diagonsis, 31st Annual International Conference of the IEEE EMBS Minneapolis, pp 1453-1456, 2009.

[15] Y.P. Kee, I. Lila Iznita, M.H. Ahmad Fadzil, A.N. Hanung, N. Hermawan and S.A. Vijanth, Conference on Innovative Technologies in Intelligent Systems and Industrial Applications (CITISIA 2009), 2009.

[16] V. Bevilacqua, S. Camb`o, L. Cariello, and G. Mastronardi, A Combined Method to Detect Retinal Fundus Features, European Conference on Emergent Aspects in Clinical Data Analysis, Pisa, Italy, Sept. 2005.

[17] P. Aravindhan and P.N. Jebarani Sargunar, Automatic Exudates Detection in Diabetic Retinopathy Images Using Digital Image Processing Algorithms, Proceedings of the Int. Conf. on Information Science and Applications ICISA 2010, pp 26-30, February, 2010.

[18] I.K.E. Purnama, K.Y.E. Aryanto. Branches Filtering Approach to Extract Retinal Blood Vessels in Fundus Image,

[19] L. Gagnon, M. Lalonde, M. Beaulieu, and M. C. Boucher, Procedure to detect anatomical structures in optical fundus images, Proc. Conf. Med. Imag. 2001, Image Processing (SPIE 4322), San Diego, pp.1218-1225, 2001.

[20] A. F. M. Hani and H. A. Nugroho, Model-Based Retinal Vasculature Enhancement in Digital Fundus Image using Independent Component Analysis, IEEE Symposium on Industrial Electronics and Applications (ISIEA 2009), pp 160-164, 2009.

[21] A. Aquino, M. E. Gegúndez-Arias and D. Marín, Detecting the Optic Disc Boundary in Digital Fundus Images Using Morphological, Edge Detection, and Feature Extraction Techniques, IEEE TRANSACTIONS ON MEDICAL IMAGING, (29), 1860-1869, Nov. 2010.

[22] E. N. Bruce, Biomedical Signal Processing and Signal Modeling, Willey Press, 2000.

[23] S. L. Marple, Digital Spectral Analysis with Applications, Prentice Hall, Inc., 1987.

[24] M. H. Hayes, Statistical Digital Signal Processing and Modeling, John Wiley and Sons, New York, 1996

[25] J. G. Proakis and D.G. Manolakis, Digital Signal Processing: Principles, Algorithms and Applications, 4th ed., Pearson Prentice Hall, 2007.

[26] D.G. Manolakis, V. K. Ingle and S. M. Kogon, Statistical and Adaptive Signal Processing, Mc. Graw Hill, 2000.

[27] M. Aibinu, M. Nilsson, M. J. E. Salami and A. A. Shafie, Voice Activity Detection Using Modeling Approach submitted for publication in Computers in Medicine and Biology, 2010.

[28] Y. H. Hu and J Hwang, Introduction to Neural Networks for Signal Processing, CRC Press LLC, 2002.

[29] S. Haykin, Neural Networks: A comprehensive foundation, 2nd ed., Eaglewood, Cliffs, NJ: Prentice Hall. 
[30] A. M. Aibinu, M. J. E. Salami, and A. A. Shafie, Determination of Complex-Valued Parametric Model Coefficients Using Artificial Neural Network Technique, AANS, volume 2010 (2010), Article ID 984381

[31] A. Hirose, Complex-Valued Neural Networks, Series on Studies in Computational Intelligence, pp. 176, ISBN-10: 3-540-33456-4, ISBN-13: 978-3-540-33456-9, New York, NY- Springer-Verlag, 2006.

[32] A. Hirose (Editor), Complex-Valued Neural Networks, Theories and Applications, Series on Innovative Intelligence, pp. 363, ISBN-981-238-464-2, 2003.

[33] A. Hirose. Complex-valued neural networks for more fertile electronics, Journal of the the IEICE, 87(6):447-449, June 2004.

[34] A. Hirose, Complex-valued neural networks: The merits and origin, Proceedings of Int. Joint. Conf. on Neural Networks pp- 257-264, June, 2009.

[35] A. Bhuiyan, B. Nath, K. Ramamohanarao, R. Kawasaki and T. YinWongth, Automated Analysis of Retinal Vascular Tortuosity on Color Retinal Images, Science+Business Media, LLC 2010, 25 May 2010.

[36] F. Villalobos and E. Riveron, Fast Automatic Retinal Vessel Segmentation and Vascular Landmarks Extraction Method for biometric Applications', International Conference on Biometrics, Identity and Security (BIdS), 2009, pp 1-10, 22-23 Sept. 2009

[37] The DRIVE database, Image Sciences Institute, University Medical Center Utrecht, The Netherlands /http://www.isi.uu.nl/Research/Databases/DRIVE/S, last accessed on 30th April, 2009.

[38] T. Fawcett, ROC Graphs: Notes and Practical Considerations for Researchers, Technical Report MS 1143 - Extended version of HPL-2003-4, HP Laboratories; 2004. OpenURL. 